\title{
Chemical Imaging of Direct Compression Pharmaceutical Formulations
}

\author{
J.P. Neilly, A.D. Vogt, M.J. Pheil, and T.S. McDermott
}

Abbott Laboratories, Global Pharmaceutical Research and Development, Department R4R9, AP31, 200 Abbott Park Rd., Abbott Park, IL 60064-6202

Solid oral formulations are complex mixtures of active pharmaceutical ingredients (API) and excipients. Excipients are utilized for a verity of functions such as diluent, disintegrent, lubricant, binder or glidant. Thus control and monitoring of all ingredients in a solid formulation is critical to manufacturing a quality product. Chemical imaging has been recently used to characterize chemical distribution of ingredients in tablets [2] and on tooling [3] to study sticking and picking phenomena. In this study, chemical imaging was used to monitor the distribution of chemical components in solid oral formulations.

Two chemical imaging techniques were used to monitor the distribution of the drug substance and several key excipients during formulation blending and tablet compression. Scanning electron microscopy (SEM) combined with elemental mapping by energy dispersive x-ray spectroscopy (EDS) was used to show the distribution of formulation components with unique elements (heteroatoms). Dispersive Raman spectroscopy was also used to show the distribution of organic formulation components. A component must be Raman active and have a unique Raman shift to be positively identified in a formulation. Since Raman spectroscopy is sensitive to organic materials while EDS works well with components containing inorganic elements, the two techniques often provide complimentary data.

Direct compressed tablets have surfaces suitable for chemical imaging and were imaged directly with little sample preparation. Particles and powders have rough topography and thus were compressed into flat discs using a hand operated press typically used to prepare samples for infrared spectroscopy. Discs and tablets were mounted on SEM stubs and examined by SEM and Raman microscopy. All SEM samples were examined in environmental mode, thus eliminating the use of conductive coatings.

The EDS silicon maps in Figure 1 clearly showed that high shear blending eliminated aggregates of silicon dioxide which were initially present. Raman images of the blend were also generated using unique bands in the Raman spectrum corresponding to the drug substance and several excipients. The Raman images in Figure 2 clearly show that high shear blending distributes the API within the excipient matrix compared with the initial matrix before blending. Silicon dioxide has a very weak Raman signal thus was not able to be detected in this experiment.

Chemical imaging by a combination of SEM/EDS and Raman Microscopy can clearly show the distribution of the drug substance and major excipients in solid formulations. The two techniques can be complimentary and can provide valuable insight during formulation development.

References

[1] A.D.Vogt, et al., Microsc. Microanal. 15 (Suppl. 2) (2009) 380.

[2] J. P. Neilly, et al., Microsc. Microanal. 15 (Suppl. 2) (2009) 18. 

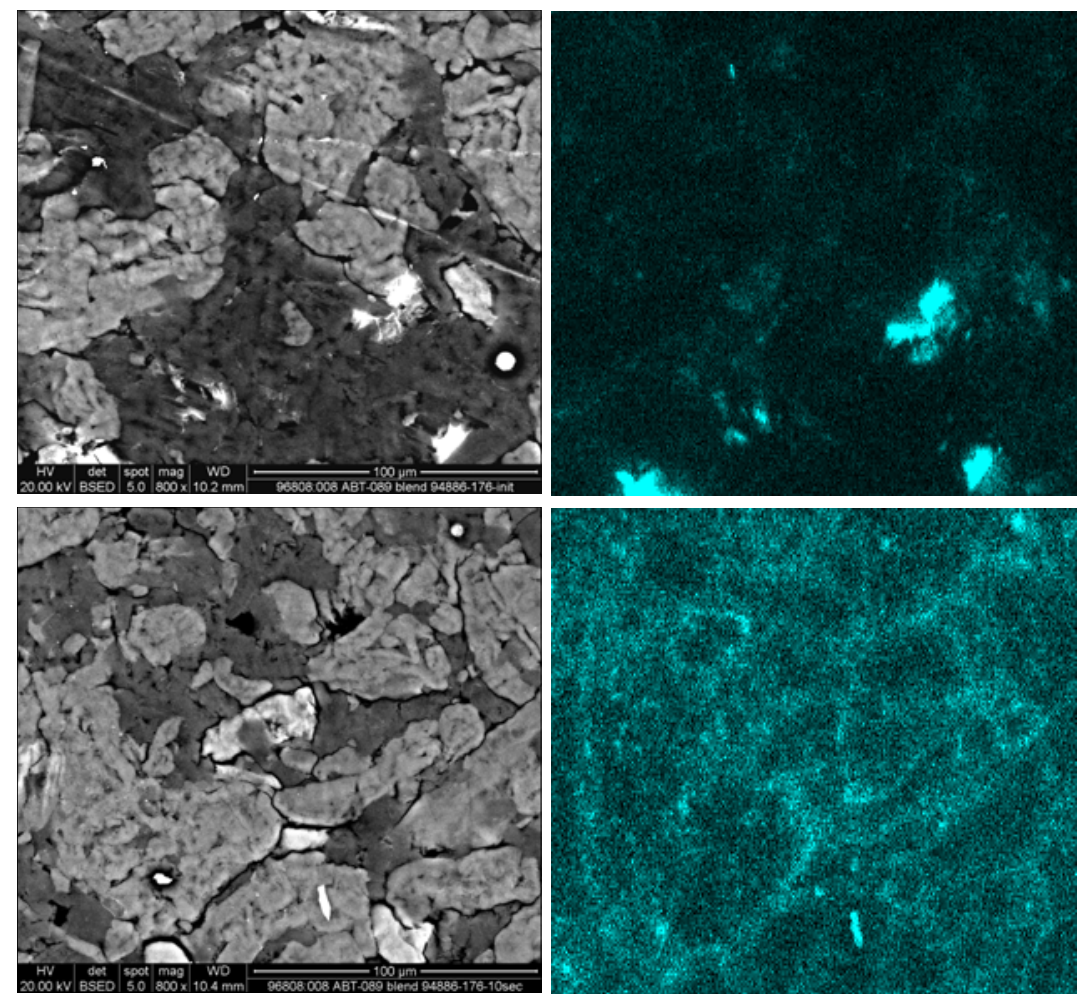

FIG. 1. SEM images (left) and corresponding silicon elemental maps of tablet blend before high shear blending (top row) and after 10 seconds of high shear blending (bottom row).
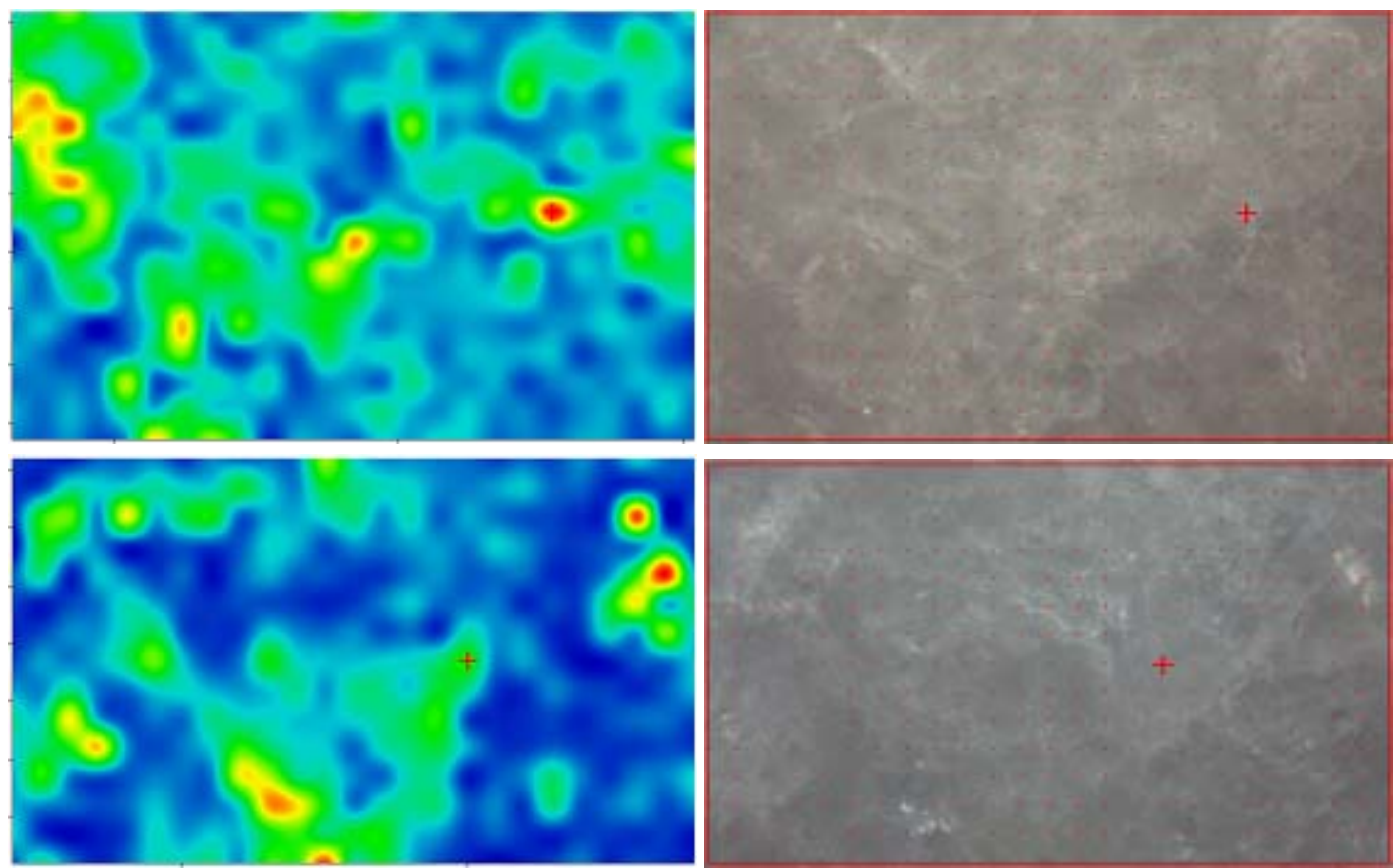

FIG. 2. Raman chemical images corresponding to the $771 \mathrm{~cm}^{-1}$ shift band of the drug substance (left) and corresponding video images (right) in the tablet blend before blending (upper images) and after 10 seconds of high shear blending (lower images). Image width equal $125 \mu \mathrm{m}$. 